\title{
ANALYSIS OF ALBUMINS, USING ALBUMIN BLUE 580, BY CAPILLARY ELECTROPHORESIS AND LASER- INDUCED FLUORESCENCE
}

\author{
Wei-Lung Tseng, Tai-Chia Chiu, Jang-Mao Weng, and \\ Huan-Tsung Chang* \\ Department of Chemistry, National Taiwan University, \\ Taipei, Taiwan, R. O. C.
}

\begin{abstract}
We report a new method for analysis of albumins with albumin blue 580 (AB 580) by capillary electrophoresis (CE) using a relatively low-cost He-Ne laser. During separation, albumins and $A B$ 580 formed complexes bearing intensive fluorescence in Trisborate (TB) buffers. The analysis of human serum albumin (HSA) was fast (4 min); reproducible (relative standard deviation (RSD) values of the migration time and peak height were less than $1 \%$ and $2 \%$, respectively); and sensitive (the limit of detection at signal-to-noise ratio $=3$ was $11 \mathrm{nM}$ ). Compared to other common dyes, such as bromphenol blue and merocyanine 540 for detecting albumins, AB 580 provides advantages of low fluorescence background, stability, sensitivity, and selectivity.

Without sample pretreatment, this proposed method was employed to determine HSA in urine and blood cells from a normal male, with results of $5.2 \pm 0.2 \mathrm{mg} / \mathrm{L}$ and about $8.2 \pm 0.2$
\end{abstract}

*Corresponding author. E-mail: changht@mail.ch.ntu.edu.tw 
$\mathrm{zmol} / \mathrm{cell}$. To further increase the resolving power of this method, the tryptic digest of HSA was separated using 0.6\% PEO. From the fact that only two peaks were shown in the electropherogram, we suggested that HSA and AB 580 formed 1:1 complexes. This method also allowed for rapid separation of HSA, deoxyribonuclease I, and their complexes.

\section{INTRODUCTION}

The quantitative determination of albumins in biological fluids is of importance in diagnosis and in many biomedical sciences. Variations in the concentration of HSA in body fluids are associated positively with a number of diseases, such as renal disease, diabetes, and liver disease.(1-3) However, it is difficult to precisely determine the concentration of albumins in biological fluids, mostly due to complicated matrices and sometimes low amounts. For example, the normal concentrations of HSA in cerebrospinal fluids and urine are around 200 $\mathrm{mg} / \mathrm{L}$ and less than $25 \mathrm{mg} / \mathrm{L}$, respectively. For detecting low concentration of albumins, immunoassay and fluorescence assay have emerged to minimize interference from other proteins for many years.(4-6)

With advantages of rapidity, high-resolving power, and sensitivity, capillary electrophoresis with laser-induced fluorescence (CE-LIF) has become one of the most powerful techniques for analysis of a variety of biological samples.(7-9) In particular, for the past few years, there has been increasing interest in the use of CE-LIF for protein analysis.(10-12) Analysis of proteins containing residues of tryptophan, tyrosine, and phenylalanine is feasible in CE by using an expensive argon ion laser (275-nm line),(13) and the limit of detection (LOD) at a signal-to noise ratio $=3(\mathrm{~S} / \mathrm{N}=3)$ for BSA down to $\mathrm{nM}$ has been reported.(14) In addition, relatively economical, compact, pulsed $\mathrm{KrF}$ excimer laser (248-nm line) has been employed for the same purpose.(15) However, for optimum sensitivity, the analysis of proteins with characteristics of intrinsic fluorescence is generally carried out at high $\mathrm{pH}$, which may induce changes in conformation and activity.(16) To prevent these problems, CE-LIF has been commonly applied to analysis of proteins derivatized with fluorophores such as fluorescein-5-isothiocyanate by using argon ion lasers.(17)

Usually, attention must be paid to minimize interferences from byproducts and problems like sensitivity associated with incomplete reactions when conducting derivatization. To minimize problems related to denaturation of proteins and/or interferences from byproducts, it is essential to perform analysis under mild conditions using relatively low-cost lasers and suitable dyes. Recently, incorporation of long-wavelength lasers, such as He-Ne lasers, has been given 
considerable attention for analysis of proteins labeled with fluorophores such as b-phycoerythrin, 5-furoylquinoline-3-carboxaldehyde, and indocyanine green (18-20) in CE. The principle advantages using He-Ne lasers for analysis of proteins include: compact, inexpensive, stable output, low matrix interference, and a low risk in destroying samples.

In spite of high-resolving power, analysis of complex biological samples, such as urine and blood by CE-LIF, is sometimes not satisfied.(21,22) In this regard, use of dyes with high selectivity for analytes of interest is one of the favorable means. For example, albumin blue 580 (AB 580) has been used for analysis of HSA because it binds quickly to albumin in a specific way to undergo dramatic fluorescence enhancement. Although, AB 580 has been described for highly sensitive determination of albumins (LOD for HSA is about 0.4 $\mathrm{mg} / \mathrm{L}),(23)$ interference from complicated sample matrices may cause a significant error in trace analysis of albumin. $(4,24)$ Thus, our goal was to develop a simple method for sensitive analysis of HSA in biological samples using CE with a He-Ne laser. The simple developed method was also employed to explore the interactions between AB 580 and HSA, and between deoxyribonuclease (DNase I) and HSA, respectively.

\section{EXPERIMENTAL}

\section{Chemicals and Biochemicals}

Reagent-grade tris(hydroxymethyl)aminomethane (Tris), $\mathrm{H}_{3} \mathrm{BO}_{3}$ and $\mathrm{NaOH}$ used for preparing Tris-borate (TB) buffers, $\mathrm{pH} 8.5$ and 10.0, respectively, and poly(ethylene oxide) (molecular weight $=8,000,000$ ) were obtained from Aldrich (Milwaukee, WI, USA). Human serum albumin (HSA), bovine serum albumin (BSA), sheep serum albumin (SSA), trypsin, DNase I, bromphenol blue (BPB), Merocyanine 540 ( $\mathrm{MC} 540$ ), $\mathrm{NaCl}, \mathrm{NaH}_{2} \mathrm{PO}_{4}, 2$-isopropanol, and dimethyl sulfoxide (DMSO) were from Sigma (St. Louis, MO, USA). AB 580 was from Molecular Probe (Eugene, OR, USA).

\section{Solutions}

The concentrations of $\mathrm{AB} 580$ and $\mathrm{BPB}$ stock solutions prepared in 2-isopropanol were both $10 \mathrm{mM}$, while that of MC 540 prepared in DMSO was $1 \mathrm{mM}$. They were stored at $4{ }^{\circ} \mathrm{C}$ and used for analysis of proteins after dilution with TB buffers. The phosphate buffered saline (PBS), $\mathrm{pH} 7.4$, used for washing cells was prepared from dissolving 8.0-g NaCl, 0.2-g $\mathrm{KH}_{2} \mathrm{PO}_{4}, 0.46-\mathrm{g} \mathrm{Na} \mathrm{HPO}_{4}$, and 0.2-g 
$\mathrm{KCl}$ in.1-L $\mathrm{H}_{2} \mathrm{O}$. PEO solutions were prepared either in 40 or $200 \mathrm{mM}$ TB buffers, $\mathrm{pH}$ 8.5.(25)

\section{Sample Preparation}

After centrifugation of the blood in EDTA from a normal male at a speed of $1000 \mathrm{rpm}$ for $10 \mathrm{~min}$, the supernatant was removed and fresh wash PBS buffer was added. The process was repeated several times for the plasma removal factor of 106. Prior to analysis, cells were lysed with 1-mL TB buffer using ultrasonic means for $20 \mathrm{~min}$. Urine from a normal male, diluted with the TB buffer (v/v $1: 1$ ), was injected into the capillary for analysis without any pretreatment. The digestion of $1 \mathrm{mM}$ HSA was carried out in $0.5 \mathrm{nM}$ trypsin aqueous solution, $\mathrm{pH}$ 8.5 , at $37^{\circ} \mathrm{C}$ overnight. HSA and DNase I were mixed at a ratio of $1: 15$ and reacted at ambient temperature for specific times.

\section{Instrumentation}

The basic design of the separation system has been previously described.(25) Briefly, a high-voltage power supply (Gamma High Voltage Research Inc., Ormond Beach, FL, USA) was used to drive electrophoresis. The entire detection system was enclosed in a black box with a high-voltage interlock. High-voltage end of the separation system was put in a home-made plexiglas box for safety. A $1.5-\mathrm{mW}$ He-Ne laser with 594-nm output from Melles Griot (Irvine, CA, USA) was used for excitation when BPB and AB 580 were used. The light was collected with a $10 \mathrm{X}$ objective (numerical aperture. $=0.25$ ). One $610-\mathrm{nm}$ interference filter was used to block scattered light before the emitted light reaches the phototube (R928 from Hamamatsu, Shizuoka-Ken, Japan). When using MC 540, a 1.5-mW He-Ne laser with 543-nm output and a 580-nm cutoff filter were used. On the other hand, when detecting the intensity of intrinsic fluorescence of proteins, a pulsed Nd:YAG laser at $266 \mathrm{~nm}$ and a UG1 filter were used. The amplified signals were transferred directly through a $10-\mathrm{kW}$ resistor to a 24-bit A/D interface at $10 \mathrm{~Hz}$ (BorwinTM, JMBS Developments, Le Fontanil, France) and stored in a PC.

\section{Separation}

Separations were performed in 40-cm capillaries (Polymicro Technologies, Phoenix, AZ, USA), $75 \mathrm{~mm}$ i.d. and $365 \mathrm{~mm}$ o.d. Prior to analysis, the capillaries (effective length: $30 \mathrm{~cm}$ ) were equilibrated with $0.1 \mathrm{M} \mathrm{NaOH}$ overnight, and 
washed with TB buffer for at least $10 \mathrm{~min}$. Injections were performed hydrodynamically at $22.5-\mathrm{cm}$ height for $4 \mathrm{~s}$, and separations were performed at $15 \mathrm{Kv}$.

\section{RESULTS AND DISCUSSION}

\section{Sensitivity}

AB 580 molecules are anionic and not fluorescent, although they quickly become intensive fluorophores after binding with albumins. The maximum excitation wavelength and emission wavelength of the complex are around 593 and $608 \mathrm{~nm}$, respectively. These characteristics make it quite suitable for on-column analysis of HSA in C,E using a relatively low-cost He-Ne laser. During separation, HAS with slightly higher electrophoretic mobility toward the anode, traversed AB580, migrating from the buffer reservoir in the anode end and, subsequently, formed complexes. Because electroosmotic flow (EOF) was higher than the electrophoretic mobility of HSA complexes, the analytes were detected near the cathode end. The electropherogram of $60 \mathrm{nM}$ HSA is shown in Fig. 1. From the peak height and noise, the LOD $(\mathrm{S} / \mathrm{N}=3)$ for HSA was about $11.1 \mathrm{nM}$. Although the LOD was higher than that (ca. $5 \mathrm{nM}$ ) measuring with a fluorome-

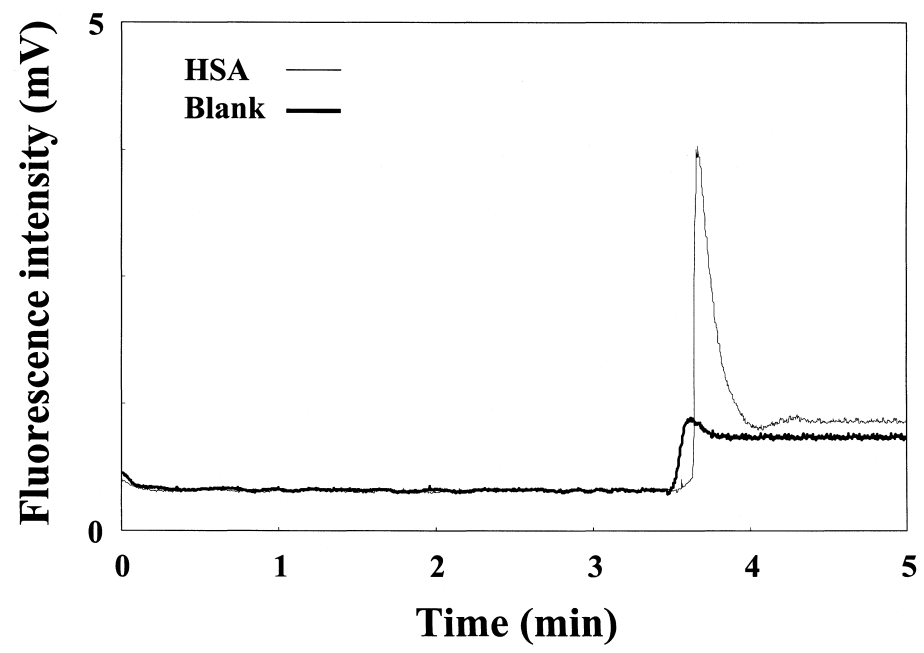

Figure 1. Detection of $60 \mathrm{nM}$ HSA in $40 \mathrm{mM}$ TB buffer, $\mathrm{pH} 8.5$, containing $1 \mathrm{mM}$ AB580 by CE-LIF. Conditions: capillary filled with $40 \mathrm{mM}$ TB buffer; total length $=40$ $\mathrm{cm}$; effective length $=30 \mathrm{~cm}$; hydrodynamic injection: $22.5 \mathrm{~cm}$ for $4 \mathrm{~s}$; run voltage $=15$ $\mathrm{kV}$. 
ter, $(4,23)$ the injection amount was only several tens of attomole (several $\mathrm{nL}$ of injection volume).

The baseline, slightly shifted after the peak corresponding to HSA complexes, was due to the small fluorescence background from AB 580 that was not filled in the capillary prior to analysis. A small occuring bump, shown in a blank test, was due to the concentration gradient near the interface between TB buffer and TB buffer containing AB 580. Table 1 shows the LOD values for HSA, BSA, and SSA are 11.1, 13.3, and $22.1 \mathrm{nM}$, respectively. The fluorescence intensity increased linearly with the concentration of the three proteins over the range of $60 \mathrm{nM}$ to $6 \mathrm{mM}$. With such high sensitivity and a good linearity, this method should have the potential for analysis of HSA in biological samples.

\section{Interferences}

With a high resolving power, $\mathrm{CE}$ has become one of the most powerful tools for analysis of biological samples, such as plasma and urine. Thus, when HSA analysis was performed using AB 580 in CE, problems associated with matrices should not be pronounced compared to that with a fluorometer. To test this suggestion, we analyzed samples containing $10 \mathrm{mM}$ common interferences, including pepsin, trypsin lactoglobulin, urea, and uric acid.(4,24) The recovery for $0.1 \mathrm{~m} \mathrm{M} \mathrm{HSA}$ is in the range of 98 to $102 \%$. Our reasoning for good recoveries is that the electrophoretic mobility values of HSA and interferences are different and $\mathrm{AB} 580$ is very specific for HSA. Interferences may also come from other small molecules that bind to HSA in many types.(26) Most strongly bound are hydrophobic organic anions of medium size, 100-600 g/mol, such as palmitate acid (binding constant: $1.5 \times 10^{8}$ ). Tryptophan is less hydrophobic and small, which is held less strongly (binding constant: $1.0 \times 10^{4}$ ) but still specific.

Table 1. LOD and Linear Regression Calculations for Albumins in CE Using $10 \mathrm{mM} \mathrm{AB}$ 580

\begin{tabular}{lcccc}
\hline Proteins & LOD $(\mathrm{nM})$ & Slope $(\mathrm{A})$ & Intercept $(\mathrm{B})$ & $\mathrm{r}^{*}$ \\
\hline HSA & 11.1 & 88.8 & 401.9 & 0.983 \\
BSA & 13.3 & 96.0 & 51.6 & 0.992 \\
SSA & 22.1 & 107.8 & 119.1 & 0.979 \\
\hline
\end{tabular}

${ }^{*} \mathrm{r}$ is the linear regression coefficient. The concentration (y) of albumins could be calculated using $\mathrm{y}=\mathrm{Ax}+\mathrm{B}$, where $\mathrm{x}$ represents the peak height for albumins. The concentration ranges of albumins determined were $60 \mathrm{nM}$ to $6 \mathrm{mM}$. 
To reveal the effects of small molecules on determining HSA using AB 580 , we added $10 \mathrm{mM}$ palmitate acid and tryptophan to the sample containing 0.5 mM HSA and performed the separation. A slight variation in the migration time (results not shown) was observed, indicating that these acids did bind to HSA. Unfortunately, we could not separate HSA complexes that did and did not contain fatty acids under this separation condition. Since the fluorescence intensity of HSA complexes did not change, we suggested that AB 580 might bind in a different site and the existence of these acids did not cause serious problems on detecting HSA.

\section{Comparison of AB580, BPB, and MC 540}

To further explore the characteristics of high selectivity and sensitivity of AB 580 for analysis of HSA, it is instructive to determine the binding constant. We measured the fluorescence intensities of the complexes formed between constant amounts of albumins and different amounts of AB 580. From the slope of the Scatchard plot (not shown), the calculated binding constant was $4.2 \times 10^{7}\left(\mathrm{R}^{2}\right.$ $=0.992$ ), providing that 1:1 complexes formed. As shown in Table 2, AB 580 formed more stable complexes with HSA compared to those using BPB, and MC 540 , other common dyes used for determination of HSA. As expected, the LOD values for HSA were higher using BPB and MC 540, which were 89.2 and 183.9 $\mathrm{nM}$, respectively. Table 2 also shows that the reproducibility of quantitative results (peak height) using $\mathrm{AB} 580(\mathrm{RSD}=1.87 \%)$ was better than those using $\mathrm{BPB}(\mathrm{RSD}=3.10 \%)$ and $\mathrm{MC} 540(\mathrm{RSD}=2.56 \%)$, presumably because of the adsorption of BPB and MC540 on the capillary wall. It is well accepted, that the adsorption of solutes on the capillary wall is more profound for hydrophobic compounds,(27) leading to decreases in EOF and poor reproducibility. As a result, the RSD values of the migration time of HSA complexes using BPB and

Table 2. Comparison of the Analysis of HSA by Different Methods

\begin{tabular}{lccccccl}
\hline Dyes & $\lambda_{\text {ex }}(\mathrm{nm})$ & $\lambda_{\text {em }}(\mathrm{nm})$ & LOD $(\mathrm{nM})$ & $\operatorname{RSD}(\%)^{\mathrm{a}}$ & $\mathrm{K}_{\mathrm{b}}$ & $\mathrm{N}^{\mathrm{b}}$ & \multicolumn{1}{c}{ Ref } \\
\hline AB580 & 593 & 608 & 11.1 & 1.87 & $4.2 \times 10^{7}$ & 1 & This work \\
AB633 & 620 & 635 & $-{ }^{\mathrm{c}}$ & $-{ }^{\mathrm{c}}$ & $1.3 \times 10^{7}$ & 1 & Ref. 4 \\
AB670 & 669 & 687 & $-{ }^{\mathrm{c}}$ & $-{ }^{\mathrm{c}}$ & $1.8 \times 10^{7}$ & 1 & Ref. 4 \\
BPB & 592 & 625 & 89.2 & 3.10 & $1.5 \times 10^{6}$ & 3 & This work \\
MC540 & 555 & 581 & 183.9 & 2.56 & $1.3 \times 10^{6}$ & 1 & This work \\
\hline
\end{tabular}

${ }^{a}$ Three measurements of peak height.

${ }^{b}$ Numbers of dye molecules bound to each HSA.

"Not given. 
MC540 were both $>3.0 \%$, which were higher than that using AB $580(<1 \%)$. We should also mention that MC 540 was quite unstable at high $\mathrm{pH}$. To that end, it is important to note that $\mathrm{AB} 580$ is superior over BPB and MC 540 for determination of HSA in terms of sensitivity, reproducibility, and specificity.

To examine our assumption that $\mathrm{AB} 580$ forms 1:1 complex with HSA, HSA and its tryptic digest were separately analyzed using this method. In order to achieve better resolution, the separation was carried out in $0.6 \%$ PEO. Figure 2A shows two peaks corresponding to HSA complexes, likely due to monomers and dimmers. On the other hand, Fig. 2B shows that a new peak appeared and the two peaks corresponding to HSA dramatically decreased (HSA was digested markedly) when injecting the tryptic digest. In order to identify the new peak, we injected the same amount of trypsin for analysis. No peak was observed in 20 min (not shown), indicating that AB 580 is not selective for trypsin. Thus, we concluded that the peak had to be from the tryptic digest. As reported that there are more than 79 peptide fragments in tryptic digest of HSA, we inferred that AB 580 forms 1:1 complexes with HAS.(28)

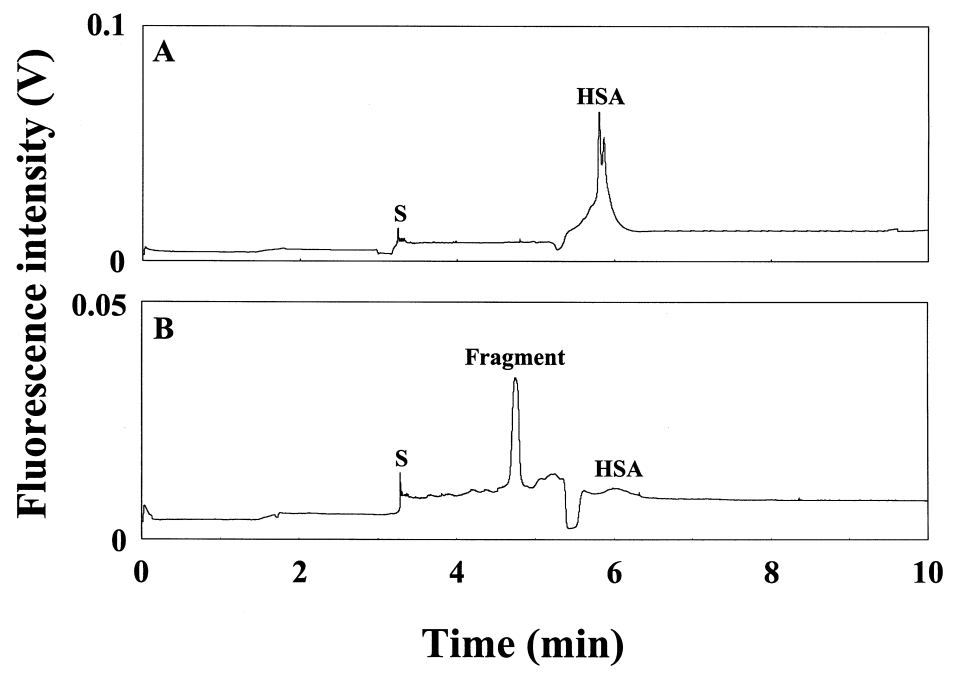

Figure 2. Separation of samples containing HSA and trypsin digest of HSA, respectively, in $0.6 \%$ PEO prepared in $40 \mathrm{mM} \mathrm{TB}, \mathrm{pH}$ 8.5. (A) 1 mM HSA. (B) trypsin digest of HSA; [HSA] $=1 \mathrm{mM}$ and [trypsin] $=5 \mathrm{nM}$. Capital $\mathrm{S}$ represents a system peak. Conditions: capillary filled with $100 \mathrm{mM}$ TB buffer, $\mathrm{pH}$ 10.0. Others conditions were as Figure 1. 


\section{Interaction Between DNase I and BSA}

It has long been known that DNase I requires divalent cations for full activity to cleave DNA with a nicking activity that is inhibited by BSA under neutral $\mathrm{pH}$ conditions. $(29,30)$ The most pronounced inhibition occurs around $\mathrm{pH}$ 4.2. Figure $3 \mathrm{~A}$ shows the separation of a sample containing BSA and DNase I with a ratio of $1: 15$ in $0.6 \%$ PEO. The peak corresponding to BSA became broader and the migration time increased with increasing the ratio of DNase I to BSA (results not shown). This is probably due to the adsorption of DNase I (glycoprotein) at high concentrations on the capillary wall. The peak in front of that corresponding to BSA only appeared at high concentrations of DNase I (10 times BSA concentration). To identify this peak, we performed the separation of DNase I under the same condition. No peak was observed, indicating that AB 580 was not selective for DNase I and the peak might correspond to the complex between BSA and DNase I.

To further support our assumption, we analyzed the sample using a Nd:YAG UV laser $(266 \mathrm{~nm})$ and the result was shown in Figure $3 \mathrm{~B}$. As the concentration of DNase I was increased (the first two peaks increased), the peak corresponding to BSA-DNase I complex increased slightly, while the peak corre-

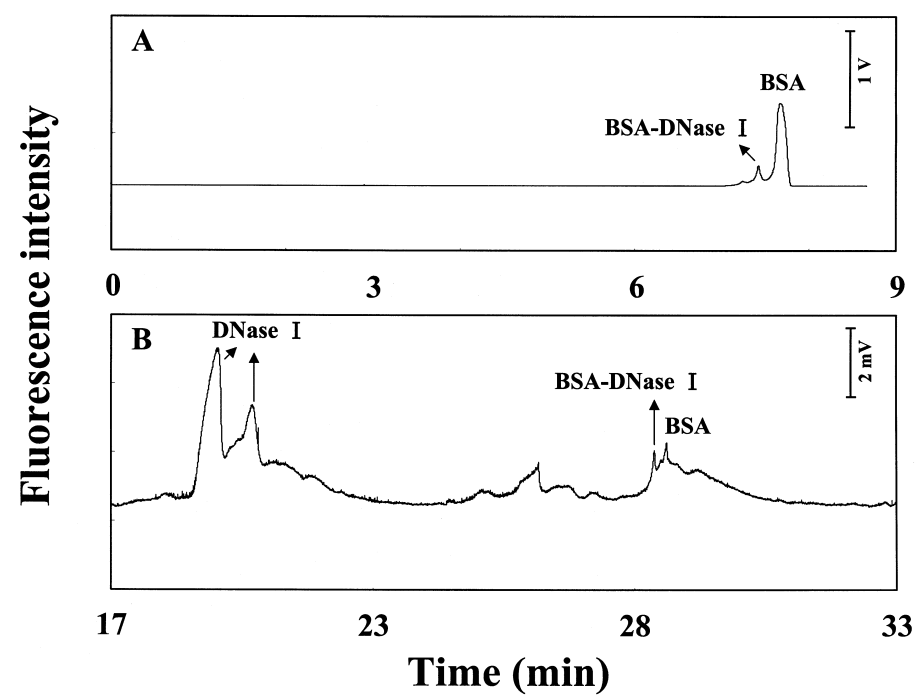

Figure 3. Separation of a sample containing $50 \mathrm{mM}$ BSA and $0.75 \mathrm{mM}$ DNase I by two different methods. (A) He-Ne laser and (B) Nd:YAG laser. Conditions: the same as Figure 2 in (A); capillary filled with $1500 \mathrm{mM}$ TB buffer, $\mathrm{pH} 10$, and buffer vials contained $0.6 \%$ PEO prepared in $200 \mathrm{mM} \mathrm{TB}, \mathrm{pH} 8.5$, in (B). 
sponding to BSA decreased slightly. The fact of a slight increase in the fluorescence intensity and the need of high concentration of DNase I, suggested that the interaction between BSA and DNase I is weak. When using high concentrations of TB buffer, EOF was small and the electrophoretic mobility of proteins was higher. As a result, the separation times shown in Fig. 3B was much longer compared to those in Fig. 3A.

\section{Urine and Blood Cells}

To test the specific and sensitive features of $A B$ 580, a 1:1 (v/v) diluted urine sample from a normal male was separated and the result was shown in Fig. 4A. The concentration of HSA in urine calculated from the peak height was 5.23 $\pm 0.15 \mathrm{mg} / \mathrm{L}(78 \pm 2 \mathrm{nM}, \mathrm{n}=5)$, which agreed with the mean concentration of HSA in urine (about $5.6 \mathrm{mg} / \mathrm{L}$ ). Figure 4B shows the separation of $15.6 \mathrm{~nL}$ of a lysed blood sample (ca. $3-\mathrm{mL}$ blood cells in $47-\mathrm{mL}$ TB buffer). Comparing this electropherogram with that of the supernatant from the last wash cycle (no peak was detected), there is no doubt that the two peaks corresponded to components in lysed cells. The concentration of HSA calculated from the peak height of the second peak is $15.0 \pm 0.7 \mathrm{mM}$. The relatively greater amount of HSA (injection dependence of variance) and other proteins (proteins adsorption on the capillary

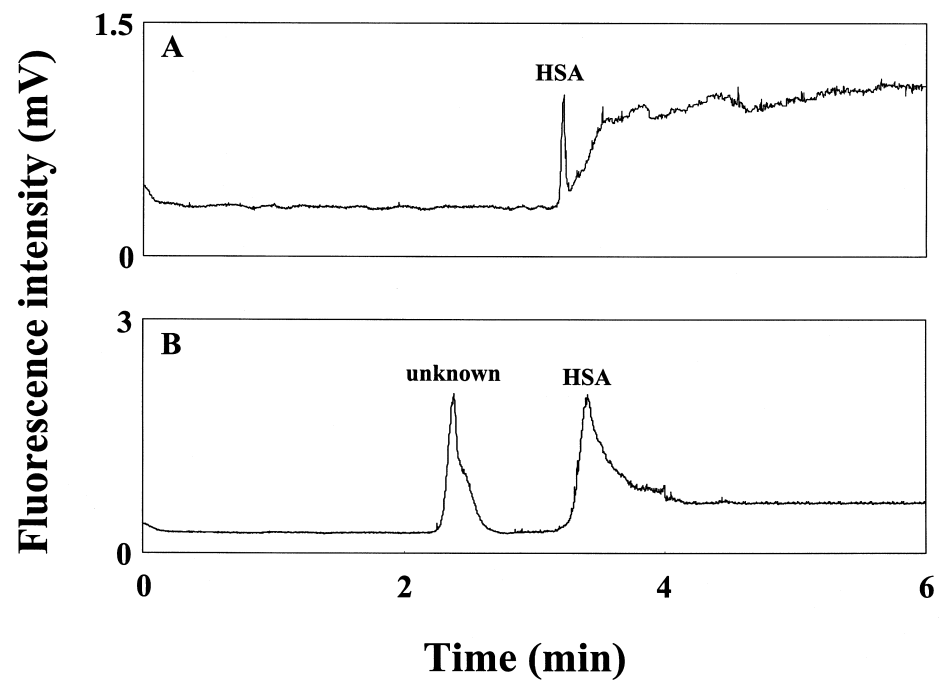

Figure 4. Analysis of HSA in urine and red blood cells using AB 580 by CE-LIF. (A) urine diluted 0.5-fold with $40 \mathrm{mM} \mathrm{TB}, \mathrm{pH} 8.5$; (B) red blood cells. Other conditions were as Figure 1. 
wall, adsorption dependence of variance) in red blood cells than that in urine is the reason for a comparably broad peak corresponding to HSA shown in Fig. 4B. If we used the volume of one blood cell equal to $84 \mathrm{fL}$, the amount of HSA in single cells was about $8.2 \pm 0.2 \mathrm{zmol}(\mathrm{n}=5)$. It should be noted that HSA might stay inside the cells or be strongly adsorbed in the membrane.

The other peak may be due to other proteins or small solutes that directly bind to $\mathrm{AB} 580$ or have strong interactions with HSA. The fact that two peaks were obtained from lysed cells suggests that it is more precise for the analysis HSA in blood samples by CE than conventional means. The fact that only one peak and two peaks found in urine and red blood cells observed again supported that this method is very specific for HSA.

\section{CONCLUSIONS}

We have taken the advantages of high selectivity and sensitivity of AB 580 for HSA to develop a relatively low-cost CE-LIF method for analysis of albumins in biological samples. Compared to BPB and MC 540, AB 580 is a better probe for albumins in terms of sensitivity, selectivity, and reproducibility. This method allow us to separate the tryptic digest of HAS, and two peaks found in the electropherogram implies that AB 580 and HSA form 1:1 complexes. Because of good reproducibility, high sensitivity, small samples injected, specificity, and rapidity, this method has the potential for evaluation of microaluminuria, which is important for early diagnosis of renal disease clinics. With high resolving power of $\mathrm{CE}$ and high specificity of $\mathrm{AB} 580$ for albumins, this method will also be applied to study the interactions between other proteins or drugs and albumins.

\section{ACKNOWLEDGMENT}

This work was supported by the National Science Council of the Republic of China under contract number NSC 89-2113-M-002-062.

\section{REFERENCES}

1. Ballmer, P.E.; Weber, P.K.; Roy-Chaudhury, P.; McNurlan, M.A.; Watson, H.; Power, D.A. Kidney Int. 1992, 41, 132-138.

2. Jefferson, L.S.; Liao, W.S.; Peavy, D.E.; Miller, T.B.; Appel, M.C.; Taylor, J.M. J. Biol. Chem. 1983, 258, 1369-1375.

3. Eppel, G.A.; Nagy, S.; Jenkins, M.A.; Tudball, R.N.; Daskalakis, M.; Balazs, N.D.H.; Comper, W.D. Clin. Biochem. 2000, 33, 487-494.

4. Kessler, M.A.; Wolfbeis, O.S. Anal. Biochem. 1992, 200, 254-259. 
5. Heegaard, N.H.H.; Kennedy, R.T. Electrophoresis 1999, 20, 3122-3133.

6. Parsons, M.P.; Newman, D.J.; Newall, R.G.; Price, C.P. Clin. Chem. 1999, 45, 414-417.

7. Tao, L.; Kennedy, R.T. Electrophoresis 1997, 18, 112-117.

8. Krull, I.S.; Deyl, Z.; Lingeman, H. J. Chromatogr. B 1994, 659, 1-17.

9. Pinto, D.M.; Arriaga, E.A.; Sia, S.; Li, Z.; Dovichi, N.J. Electrophoresis 1995, 16, 534-540.

10. Asermely, K.E.; Broomfield, C.A.; Nowakowski, J.; Courtney, B.C.; Alder, M. J. Chromatogr. B 1997, 695, 67-75.

11. Wan, Q.-H.; Le, X.C. Anal. Chem. 2000, 72, 5583-5589.

12. Hunt, G.; Nashabeh, W. Anal. Chem. 1999, 71, 2390-2397.

13. Chang, H.-T.; Yeung, E.S. Anal. Chem. 1993, 65, 2947-2951.

14. Lee, T.T.; Yeung, E.S. J. Chromatogr. 1992, 595, 319-325.

15. Chan, K.C.; Janini, G.M.; Muschik, G.M.; Issaq, H.J. J. Liq. Chromatogr. 1993, 16, 1877-1890.

16. Gianazza, E.; Galliano, M.; Miller, I. Electrophoresis 1997, 18, 695-700.

17. Wise, E.T.; Singh, N.; Hogan, B.L. J. Chromatogr. A 1996, 746, 109-121.

18. Pinto, D.M.; Arrigaga, E.A.; Craig, D.; Angelova, J.; Sharma, N.; Ahmadzadeh, H.; Dovichi, N.J. Anal. Chem. 1997, 69, 3015-3021.

19. Baars, M.J.; Patonay, G. Anal. Chem. 1999, 71, 667-671.

20. Moody, E.D.; Viskari, P.J.; Colyer, C.L. J. Chromatogr. B 1999, 729, 55-64.

21. Schmerr, M.J.; Jenny, A.L.; Buligin, M.S.; Miller, J.M.; Hamir, A.N.; Cutlip, R.C.; Goodwin, K.R. J. Chromatogr. A 1999, 853, 207-214.

22. Tseng, W.L.; Chang, H.-T. Anal. Chem. 2000, 72, 4805-4811.

23. Kessler, M.A.; Meinitzer, A.; Wolfbeis, O.S. Anal. Biochem. 1997, 248, 180-182.

24. Kessler, M.A.; Meinitzer, A.; Petek, W.; Wolfbeis, O.S. Clin. Chem. 1997, 43, 996-1002.

25. Chen, H.-S.; Chang, H.-T. Anal. Chem. 1999, 71, 2033-2036.

26. Richieri, G.V.; Anel, A.; Kleinfeld, A.M. Biochemistry 1993, 32, 75747580 .

27. Ding, W.; Fritz, J.S. Anal. Chem. 1998, 70, 1859-1865.

28. Fisichella, S.; Foti, S.; Maccarrone, G.; Saletti, R. J. Chromatogr. A 1995, 693, 33-44.

29. Lesca, P.; Paoletti, C. Biochemistry 1969, 64, 913-919.

30. Eshima, N.; Muta, A.; Anai, M. J. Biochem 1983, 94, 345-352. 
Copyright $\odot 2002$ EBSCO Publishing 